\title{
A Theoretical Method to determine unstressed off-rate from multiple bond force spectroscopy
}

\author{
V.K. Gupta and C.D. Eggleton \\ University of Maryland Baltimore County, Baltimore, Maryland 21250, USA
}

\begin{abstract}
Using dynamic force spectroscopy to measure the kinetic off-rates of intermolecular bonds currently requires the isolation of single molecules. This requirement arises in part because no tractable analytic method for determining kinetic off-rates from the rupture of a large number of bonds under dynamic forces is currently available. We introduce a novel method for determining the unstressed off-rate from dynamic force spectroscopy experiments involving a large number of bonds. Using both the Bell and Dembo models we show that the unstressed off-rate calculated using the proposed method is in good agreement with the prescribed unstressed off-rate used in Monte-Carlo simulations of multiple bond dynamic force spectroscopy experiments given initial number of bonds (50-500) and loading rate $10^{3}-10^{6} \mathrm{pN} / \mathrm{s}$.
\end{abstract}

\section{INTRODUCTION}

Cell adhesion mediated by surface-bound receptor-ligand interactions [or so-called 2D binding] plays a pivotal role in diverse biological processes, including thrombosis [1], tumor metastasis [2], arteriosclerosis [3] and inflammation [4, 5]. Changes in cell adhesion can be the defining event in a wide range of diseases, including cancer [6], arthritis [7], osteoporosis, and atherosclerosis [8]. Under real physiological conditions cellular adhesion relies on bond clusters [9]. Cells in multicellular organisms adhere to other cells and the extracellular matrix through two-dimensional clusters spanning a number range from several (leukocyte rolling) to $10^{5}$ (mature adhesion) adhesion bonds [10]. Cellular adhesion occurs through a multitude of weak interactions [9]. Multivalent interactions play a critical role in a variety of biological processes on both cellular and molecular levels [11, 12]. Multicellular organisms perform many biological functions using multivalent interactions where multiple weak individual bonds between receptor-ligand pairs combine to produce a much stronger interaction $[11,12]$. Multivalent bonds feature prominently in a variety of biological processes such as intercellular adhesion [13] and activation of T cells [14]. Biomedical researchers also utilize multivalent binding to increase the binding time of pharmaceuticals to their targets [15].

The receptor-ligand interactions that mediate cellular adhesion are often subjected to forces that regulate their dissociation via modulating off-rates. The bond lifetime of receptor-ligand bonds and the amount of force they withstand prior to rupture is determined by the kinetic off-rate in statistical models of intermolecular binding. Currently, the kinetic off-rate for a particular intermolecular bond can be determined by measuring the bond lifetime or

\footnotetext{
() 2012 Elsevier B.V. All rights reserved.
}

Publisher's Disclaimer: This is a PDF file of an unedited manuscript that has been accepted for publication. As a service to our customers we are providing this early version of the manuscript. The manuscript will undergo copyediting, typesetting, and review of the resulting proof before it is published in its final citable form. Please note that during the production process errors may be discovered which could affect the content, and all legal disclaimers that apply to the journal pertain. 
dissociation force of single bonds under an applied external force. The bond-rupture data for many single bond measurements, $O(100)$, is used to construct a rupture force histogram. The most probable rupture force is calculated from a nonlinear least-squares fits to a probability distribution and then matched with an analytic expression of the most probable rupture force to determine the kinetic off-rate [16].

Single-bond dynamic force spectroscopy experiments and analysis require a very low frequency of successful binding events to avoid multiple attachments [17]. Single molecule force spectroscopy measurement requires manipulation of the contact area so that one molecule on each surface interacts. This is achieved by reducing the probability of bond formation on contact through the control of surface chemistry, molecular density, contact force and time [18]. These conditions lead to an attachment frequency of one in ten per touch. When attachment occurs, there can be a small possibility that multiple bonds formed. However, when the contact area and surface chemistry cannot be controlled multiple interactions may dominate [18]. The presence of multiple bond ruptures in single molecule force spectroscopy measurement might lead to deviation of the force histograms from the single bond model and consequently to the poor reproducibility of the kinetic parameters [19]. This makes the accumulation of rupture force histograms through single-bond experiments time consuming and expensive [17].

The fundamental question arises whether quantitative information relevant to the single interaction can be extracted from measurements of multiple simultaneous detachments. Various statistical methods have been developed in an attempt to elucidate the singlemolecule event from the rupture of multiple attachments [20-22]. Hukkanen et al. [23] developed double-bond microscopic model in an attempt to determine the kinetic parameters associated with bond dissociation. Further, Erdmann et al. [17] developed a theoretical model based on superposition of rupture force histograms to extract the single-bond properties from the multiple bond data. These quantitative methods consider the formation of multiple bonds $O(10)$ or less and the related experimental conditions lead to attachment frequencies of 1 in 3 (or less) per touch.

A theoretical method that enables conditions with touch adhesion frequencies approaching one and that allows for a large number of bonds rupture pulling experiment is a step in providing more efficient dynamic force spectroscopy methods enabling the routine measurement of the kinetic rate of adhesion of individuals. In this work we present a novel method for determination of the unstressed kinetic off-rate for a receptor-ligand bond from the rupture of multiple bonds, $O(100)$ during a single dynamic force measurement. Increasing the order of magnitude of initial bond formation allows the experimental conditions to be relaxed to favor bond formation, enabling attachment frequencies of 1 to 1 per touch. The proposed method requires the measurement of the number of bonds, and the length of the bonds, as a function of time. Using our model to simulate the binding and rupture of multiple bonds $[24,25]$ in response to an externally applied force we demonstrate that as few as ten multiple bond pulling experiments can be conducted in order to determine the unstressed kinetic off-rate with an error less than 5\%. Given the stochastic nature of bond rupture, it is reasonable to expect that increasing the number of individual bond ruptures used in determining kinetic off-rates will increase the accuracy of the measurement. This method for determining kinetic off-rates from multiple bond dynamic force spectroscopy may prove to more efficient since fewer pulling experiments will be required.

\section{COMPUTATIONAL METHOD}

Using a Monte Carlo technique in conjunction with both Bell and Dembo models over a relevant range of loading rates $\left(10^{3}-10^{6} \mathrm{pN} / \mathrm{s}\right)$, we simulate the response of multiple bonds 
to dynamic force spectroscopy experiments. The simulations are initiated with a large number of bonds, and the corresponding time histories of bond length and bond number under a constant loading rate for the prescribed values of bond stiffness and unstressed offrate. Our analysis assumes the bond stiffness is known. The unstressed off-rate is obtained from these data sets utilizing the proposed method and compared with the prescribed unstressed off-rate used in generating the simulation data.

In our model two beads (hard spheres) are suspended in a Newtonian fluid of density $\rho$ and viscosity $\mu$. Initially the two beads are nearly touching and are allowed to adhere by multiple (L-selectin-PSGL-1) bonds $O(100)$. We assume that these molecules are located at the same anchor point on the bead surface. As soon as the total expected number of bonds between the two beads are formed we apply an equal and opposite force $F$ on the surface of each bead along the line connecting their centers causing bond stretching and eventual bond rupture (see Fig. 1). We can write an analytical expression for time varying bond length as the two beads are pulled apart. Due to symmetry we consider only one bead in writing the following momentum balance:

$$
\rho \frac{4}{3} \pi R^{3} \frac{d^{2} x}{d t^{2}}+N \sigma x+6 \pi \mu R \frac{d x}{d t}=F(t),
$$

where $x$ is the displacement of cell center as shown in Fig. 1 and $N(t)$ is number of bonds at any time $t$. The above differential equation can be integrated numerically using the initial conditions $x(0)=x^{\prime}(0)=0$. Thus knowing $x(t)$ we can simulate the detachment of receptorligand bond as a function of loading rate using the Monte Carlo method [26]. Once all the bonds are ruptured the two beads separate from each other to a distance where further bond (re)formation is impossible and the simulation is terminated.

\section{A. Monte Carlo simulation of receptor-ligand interactions}

We model the bonds between adhesion molecules (ligand-receptor) anchored on the bead surfaces as Hookean springs, so the chemical force on each bead due to each bond, $\mathbf{F}_{\mathbf{b}}$, is given by

$$
\mathbf{F}_{\mathbf{b}}=\sigma(\mathbf{x}-\lambda)
$$

where $\sigma$ is the bond spring constant, $\mathbf{x}$ is the stressed bond length, and $\lambda$ is the equilibrium bond length. According to the Bell model the forward rate constant, $k_{f}$, is given by [10]

$$
k_{f}=k_{f}^{0} \exp \left[\frac{\sigma(x-\lambda)\left(x_{\beta}-0.5(x-\lambda)\right)}{k_{b} T}\right] \text {, }
$$

and the reverse rate constant, $k_{I}$, is given by [10]

$$
k_{r}=k_{r}^{0} \exp \left(\frac{x_{\beta} F_{b}}{k_{b} T}\right) \text {, }
$$

where $x_{\beta}$ is the reactive compliance and $F_{b}$ is the force acting on the bond. Similarly the Dembo model forward rate constant, $k_{f}$, for ligand-receptor interactions is given by [27]

$$
k_{f}=k_{f}^{0} \exp \left[-\frac{\sigma_{\mathrm{ts}}(x-\lambda)^{2}}{2 k_{b} T}\right],
$$

and the reverse rate constant, $k_{r}$, is given by [27] 


$$
k_{r}=k_{r}^{0} \exp \left[\frac{\left(\sigma-\sigma_{\mathrm{ts}}\right)(x-\lambda)^{2}}{2 k_{b} T}\right] \text {, }
$$

where $k_{f}^{0}$ and $k_{r}^{0}$ are the forward and reverse rate constants at an equilibrium distance $\lambda, T$ is the absolute temperature, $k_{b}$ is the Boltzman constant, and $\sigma_{\mathrm{ts}}$ represents the spring constant in the transition state. We use Monte Carlo simulation to incorporate the stochastic nature of ligand-receptor interactions. In the simulation during a time interval $\Delta t$, the probability $\left(P_{b}\right)$ that a ligand will bind with its complementary receptor is given by [26]

$$
P_{b}=1-\exp \left(-k_{\mathrm{f}} \Delta t\right) .
$$

Similarly the probability for bond rupture is given by [26]

$$
P_{r}=1-\exp \left(-k_{\mathrm{r}} \Delta t\right) \text {. }
$$

At each time step, the probabilities of bond formation and rupture are compared to random numbers $\left(P_{\text {ran1 } 1}\right.$ and $\left.P_{\text {ran2 } 2}\right)$ lying between 0 and $1 . P_{b}>P_{\text {ran } 1}$ indicates bond formation whereas $P_{r}>P_{\text {ran2 }}$ indicates bond rupture.

\section{B. Determination of Unstressed off-rate}

1. One Model Parameter Determination-In this section we outline a methodology to determine only unstressed off-rate $\left(k_{r}^{0}\right)$ from the multiple bond force spectroscopy data assuming the other model parameter, for example the reactive compliance $\left(x_{\beta}\right)$ in the Bell model (Eqn. (4)), is known.

Decay Without Rebinding: For the case of decay without rebinding the rate equation is given by [28, 29]

$$
\frac{d N}{d t}=-N k_{r}
$$

where $N(t)$ and $\frac{d N}{d t}$ are the number of bonds and its time derivative at any time $t$, respectively. Further, $k_{I}(t)$ is given by kinetic based models such as Bell and Dembo models. If $k_{r}(t)$ is given by Eqn. 4 (Bell model), then equating Eqn. 4 and Eqn. 9 leads to

$$
k_{r}^{0}=\frac{-\frac{1}{N(t)} \frac{d N}{d t}}{\exp \left(\frac{x_{\beta} F_{b}}{k_{b} T}\right)},
$$

and if $k_{I}(t)$ is given by Eqn. 6 (Dembo model), then equating Eqn. 6 and Eqn. 9 leads to

$$
k_{r}^{0}=\frac{-\frac{1}{N(t)} \frac{d N}{d t}}{\exp \left[\frac{\left(\sigma-\sigma_{\mathrm{ts}}\right)(x-\lambda)^{2}}{2 k_{b} T}\right]} .
$$

Thus, knowing both $N(t)$ and $x(t)$ we can calculate $k_{r}^{0}(t)$ from Eqn. 10 and Eqn. 11 which allows us determination of a time averaged unstressed off-rate, $k_{r}^{0}$ as: 


$$
k_{r}^{0}=\frac{1}{T_{\mathrm{fit}}} \int_{0}^{T_{\mathrm{fit}}} k_{r}^{0}(t) d t
$$

where $T_{\text {fit }}$ is the end point in time of the data fit on $N(t)$ or $x(t)$ as illustrated in Fig. 2 for such a data set. We fit our simulation data of $N(t)$ vs $t$ with $N(t)=A t^{5}+B t^{4}+C t^{3}+D t^{2}+$ $E t+N_{0}$ where $A, B, C, D$ and $E$ are numerical constants and $N_{0}$ is the initial number of bonds. The corresponding fit for simulation data of $x(t)$ vs $t$ is chosen of the form: $x(t)=a t^{5}$ $+b t^{4}+c t^{3}+d t^{2}+e t+x_{0}$ where $a, b, c, d$ and $e$ are numerical constants and $x_{0}$ is the equilibrium bond length at $t=0$.

Decay With Rebinding: For the case of decay with rebinding the rate equation is given by $[28,29]$

$$
\frac{d N}{d t}=-N k_{r}+k_{f}\left(N_{0}-N\right)
$$

For the Bell model the above equation leads to

$$
k_{r}^{0}=\frac{-\frac{1}{N(t)} \frac{d N}{d t}-\frac{k_{f}}{N}\left(N-N_{0}\right)}{\exp \left(\frac{x_{\beta} F_{b}}{k_{b} T}\right)} .
$$

Thus, knowing both $N(t)$ and $x(t)$ we can calculate $k_{r}^{0}(t)$ from the above Eqn. (14), which allows determination of a time averaged unstressed off-rate, $k_{r}^{0}$, from Eqn. (12) as discussed above.

2. Two Model Parameters Determination-The methodology presented above outlines an approach to determine only unstressed off-rate $\left(k_{r}^{0}\right)$ from the multiple bond force spectroscopy data assuming the other model parameter, for example the reactive compliance $\left(x_{\beta}\right)$ in the Bell model (Eqn. (4)), is known. If we are interested in determining both model parameters, say $k_{r}^{0}$ and $x_{\beta}$ in the Bell model, then we slightly modify the above methodology.

Decay Without Rebinding: In this case Eqn. (10) is rearranged to give

$$
\log \left(-\frac{1}{N(t)} \frac{d N}{d t}\right)-\log \left(k_{r}^{0}\right)=\frac{x_{\beta} \sigma x(t)}{k_{b} T} .
$$

The above equation can be integrated from $t=0$ to $t=T_{\mathrm{fit}}$ to give

$$
\overline{\mathrm{LN}}-T_{\mathrm{fit}} \log \left(k_{r}^{0}\right)=\frac{x_{\beta} \sigma}{k_{b} T} \overline{\mathrm{x}},
$$

where $^{\overline{\mathrm{LN}}}=\int_{0}^{T_{\mathrm{fit}}} \log \left(-\frac{1}{N(t)} \frac{d N}{d t}\right) d t$ and $\overline{\mathrm{x}}=\int_{0}^{T_{\mathrm{fit}}} x(t) d t$. Then, using the Levenberg-Marquardt non-linear least squares fitting program [31], the Bell model parameters $\left(k_{r}^{0}, x_{\beta}\right)$ that best fit all the multiple bond rupture data for $\overline{\mathrm{LN}} \mathrm{vs} \overline{\mathrm{x}}$ to Eqn. (15) can be found.

Decay With Rebinding: In this case Eqn. (14) is rearranged to give 


$$
\log \left(-\frac{1}{N(t)} \frac{d N}{d t}-\frac{k_{f}}{N}\left(N-N_{0}\right)\right)-\log \left(k_{r}^{0}\right)=\frac{x_{\beta} \sigma x(t)}{k_{b} T} .
$$

In this case it is not straight forward to integrate the above equation to recast into an equation similar to Eq. (15) unless one assumes that the rate $k_{f}$ for bond formation is not dependent on the distance between two beads, i.e., $k_{f}=k_{f}^{0}$. However, this would be an unrealistic assumption given in reality the rate of bond formation would be distance dependent.

\section{Model and Computational Parameters}

We simulated adhesion and detachment of two beads based on data for PMN cells using the parameter values listed in Table 1 unless stated otherwise. The center to center distance between the two beads is $7.55 \mu \mathrm{m}$. A time step of $10^{-7} \mathrm{~s}$ is used in all these simulations.

\section{RESULTS}

\section{One Model Parameter Determination}

In this section we present our results dealing with the determination of only unstressed offrate $\left(k_{r}^{0}\right)$ from the multiple bond force spectroscopy data assuming the other model parameter, for example the reactive compliance $\left(x_{\beta}\right)$ in the Bell model (Eqn. (4)), is known.

Simulated Decay Without Rebinding-We simulated detachment of two beads including only bond rupture using Eq. (9) under the applied force $F=R_{f} t$ unless stated otherwise. Fig. 2 (a) shows one data set for time variation of number of bonds, $N(t)$, obtained from micromechanical model simulation using Bell model at $R_{f}=10^{5} \mathrm{pN} / \mathrm{s}, N_{0}=$ 200 , and $k_{r}^{0}=8.6 \mathrm{~s}^{-1}$. The corresponding data set for time variation of bond length, $x(t)$, is shown in Fig. 2(b). Bond rupture is synergistically regulated by thermally induced spontaneous dissociation and occurs stochastically with a characteristic bond life-time that is the reciprocal of the unstressed off-rate [30]. However, in mechanically mediated force dissociation an applied load causes bond stretching and hence lowers the chemical activation barrier to increase the probability of bond rupture according to Eqs. 4 and 8. Thus, it is seen from Fig. 2(a) that initially bond rupture is nearly linear with time. However, once number of bonds drop to about 20 the bond rupture is non-linear with time due to increase in $x(t)$ as seen in Fig. 2(b) leading to enhanced probability in bond rupture according to Eqs. 4 and 8. Further, it is seen that there is a precipitous drop in the number of bonds from about 20 to 0 due to the same reason. The corresponding data fit is given by $N(t)=A t^{5}+B t^{4}+C t^{3}+D t^{2}$ $+E t+N_{0}$ with $A=-6426810, B=2153400, C=-243128, D=11472.3, E=-1422.04, N_{0}$ $=200$ and $x(t)=a t^{5}+b t^{4}+c t^{3}+d t^{2}+e t+x_{0}$ with $a=53903.7, b=-17545.5, c=1994.05$, $d=-88.4963, e=1.39597, x_{0}=0.07 \mu \mathrm{m}$ are also shown in Fig. 2(a) and Fig. 2(b)

respectively. We consider data only for $0<t<T_{\text {fit }}$ to calculate the time averaged unstressed off-rate, where $T_{\text {fit }}$ is dictated by the kinetic model used. For this particular data set shown in Fig. $2, T_{\text {fit }}$ is approximately $0.153 \mathrm{~s}$.

Fig. 3 shows ten data sets for $k_{r}^{0}(t)$ (dotted lines) calculated from Eq. 10 corresponding to the data obtained from micromechanical model simulation using Bell model at $R_{f}=10^{5} \mathrm{pN} / \mathrm{s}$, $N_{0}=200$, and $k_{r}^{0}=8.6 \mathrm{~s}^{-1}$. The simulated value of unstressed off-rate $\left(k_{r}^{0}=8.6 \mathrm{~s}^{-1}\right)$ is shown as solid line in the same figure. For each data set it is seen that $k_{r}^{0}(t)$ fluctuates around the simulated unstressed off-rate. We calculated the time averaged unstressed off-rate for each data set using Eqn. 12. These are 7.94, 9.92, 8.77, 7.58, 8.60, 8.48, 9.59, 8.43, 8.22 and 8.99 
$\mathrm{s}^{-1}$. This gives an averaged (over ten sets) value of approximately $8.65 \mathrm{~s}^{-1}$ with standard deviation of $0.67 \mathrm{~s}^{-1}$ as listed in the Table 2 .

We further repeated the above procedure to calculate unstressed off-rate using the Bell model at $N_{0}=200$ and $k_{r}^{0}=8.6 \mathrm{~s}^{-1}$ and varying loading rates from $R_{f}=10^{3} \mathrm{pN} / \mathrm{s}$ to $R_{f}=10^{6}$ $\mathrm{pN} / \mathrm{s}$. Our findings are listed in Table 2 . It is seen that at a given loading rate the calculated unstressed off-rate is nearly identical to the simulated unstressed off-rate (maximum error < $2.0 \%$ ). We then systematically varied the simulated unstressed off-rate from 1.6 to $8.6 \mathrm{~s}^{-1}$ and calculated the unstressed off-rate using Bell model at $N_{0}=200$ and $R_{f}=10^{5} \mathrm{pN} / \mathrm{s}$. It is seen from Table 2 that at a given simulated unstressed off-rate the calculated unstressed offrate is nearly identical to the simulated unstressed off-rate (maximum error $<3.0 \%$ ). Further we then systematically varied initial number of bonds, $N_{0}$, at a fixed $R_{f}=10^{5} \mathrm{pN} / \mathrm{s}$ and simulated unstressed off-rate $k_{r}^{0}=8.6 \mathrm{~s}^{-1}$. Once again it is seen from Table 2 that for the given $N_{0}$ the calculated unstressed off-rate is nearly identical to the simulated unstressed off-rate (maximum error $<2.0 \%$ ). However, the standard deviation increases as we decrease $N_{0}$.

We further repeated the above procedure to calculate unstressed off-rate using Bell model at $N_{0}=200$ and $k_{r}^{0}=8.6 \mathrm{~s}^{-1}$ but with a different applied load of the form:

$F=F_{0}\left[1-\exp \left(-\frac{R_{f} t}{F_{0}}\right)\right]$. This type of applied load parallels the normal loading force $\left(F=R_{f}\right.$ $t)$ initially and then for longer time $\left(t \rightarrow \infty, F \rightarrow F_{0}\right)$ it acts as a constant applied load. Our findings are listed in Table 3 for different combinations of $F_{0}$ and $R_{f}$ at a fixed initial number of bonds and simulated unstressed off-rate. Once again it is seen that the calculated unstressed off-rate is in close agreement with the simulated unstressed off-rate.

We next examined the effect of changing kinetic model to Dembo model given by Eqn. 5 and 6 on the unstressed off-rate calculation. Fig. 4 shows ten data sets for $k_{r}^{0}(t)$ (dotted lines) calculated from Eq. 11 corresponding to the data obtained from micromechanical model simulation using Dembo model at $R_{f}=10^{5} \mathrm{pN} / \mathrm{s}, N_{0}=200$, and $k_{r}^{0}=3.0 \mathrm{~s}^{-1}$. The simulated value of unstressed off-rate $\left(k_{r}^{0}=3.0 \mathrm{~s}^{-1}\right)$ is shown as solid line in the same figure. For each data set it is seen that $k_{r}^{0}(t)$ fluctuates around the simulated unstressed off-rate. We calculated the time averaged unstressed off-rate for each data set using Eqn. 12. These are 3.39, 3.50, $3.01,2.84,2.88,2.51,3.75,2.59,2.99$ and $2.61 \mathrm{~s}^{-1}$. This gives an averaged (over ten sets) value of approximately $3.01 \mathrm{~s}^{-1}$ with standard deviation of $0.39 \mathrm{~s}^{-1}$ as listed in the Table 4 . We further repeated the above procedure to calculate unstressed off-rate using Dembo model at $N_{0}=200$ and $k_{r}^{0}=3.0 \mathrm{~s}^{-1}$ and varying loading rates from $R_{f}=10^{3} \mathrm{pN} / \mathrm{s}$ to $R_{f}=10^{6}$ $\mathrm{pN} / \mathrm{s}$. Our findings are listed in Table 4 . It is seen that at a given loading rate the calculated unstressed off-rate is nearly identical to the simulated unstressed off-rate (maximum error < $3.0 \%)$. We then systematically varied simulated unstressed off-rate from 1 to $10 \mathrm{~s}^{-1}$ and calculated unstressed off-rate using Dembo model at $N_{0}=200$ and $R_{f}=10^{5} \mathrm{pN} / \mathrm{s}$. It is seen from Table 4 that at a given simulated unstressed off-rate the calculated unstressed off-rate is in close aggrement to the simulated unstressed off-rate (maximum error $<2.0 \%$ ). Further we then systematically varied initial number of bonds, $N_{0}$, at a fixed $R_{f}=10^{5} \mathrm{pN} / \mathrm{s}$ and simulated unstressed off-rate $k_{r}^{0}=3.0 \mathrm{~s}^{-1}$. Once again it is seen from Table 4 that at a given $N_{0}$ the calculated unstressed off-rate is in close aggrement to the simulated unstressed offrate (maximum error $<4.0 \%$ ). However, the standard deviation increases as we decrease $N_{0}$ implying that perhaps the presented method for unstressed off-rate calculation is best suited for large $O(100)$ initial number of bonds.

Simulated Decay With Rebinding-We simulated detachment of two beads using the Bell model for both bond formation and bond rupture, Eq (3) and Eq. (4), respectively. The 
simulations were conducted for an initial bond number $N_{0}=200$, on-rate of $k_{f}^{0}=150 \mathrm{~s}^{-1}$, and off-rate of $k_{r}^{0}=8.6 \mathrm{~s}^{-1}$, at varying loading rates from $R^{f}=10^{3} \mathrm{pN} / \mathrm{s}$ to $R_{f}=10^{6} \mathrm{pN} / \mathrm{s}$. Ten pulling simulations were done for each loading rate. This gave us simulated multiple bond force spectroscopy data that accounted for bond reformation that we will analyze using various methods to extract the unstressed off-rate, $k_{r}^{0}$.

First, we use Eq. (14), the one model parameter analysis that accounts for rebinding to calculate $k_{r}^{0}(t)$. Note, $k_{f}$ is given by Eq. (3) for the Bell model that depends on the separation distance, $x(t)$, between beads. Knowing $k_{r}^{0}(t)$ we can calculate the time averaged unstressed off-rate for each data set using Eqn. (12) as done previously. The calculated unstressed offrates as a function of loading rate are listed in Table V. It is seen that at a given loading rate the calculated unstressed off-rate is nearly identical to the prescribed unstressed off-rate in the simulations (maximum error $<1.5 \%$ ).

Second, we analyze the data set obtained through simulations that included rebinding, using Eq. (10), the one model parameter analysis that neglects rebinding to calculate $k_{r}^{0}(t)$. This allows us to isolate the effects of rebinding in the calculation of unstressed off-rate. The corresponding calculated unstressed off-rates for loading rates $R_{f}=10^{3}, 10^{4}, 10^{5}$, and $10^{6}$ $\mathrm{pN} / \mathrm{s}$ are $8.57 \pm 0.33,8.59 \pm 0.24,8.5 \pm 0.19$, and $8.53 \pm 0.30 \mathrm{~s}^{-1}$, respectively.

Comparing the respective values calculated using the single parameter method that accounts for rebinding, Eq. (14) with those calculated using the method that neglects rebinding, Eq. (10) shows that the difference is less than $1.6 \%$. The off-rates calculated neglecting rebinding are slightly lower, as one might expect, since bond reformation would slow the rate of decay. This demonstrates that effect of rebinding on the calculation of unstressed offrate is not very significant at the model parameters considered. This is attributed to the fact that rebinding is an important factor initially when the two beads are relatively close to each other (see Fig. 5). Once the separation distance between the two beads increases the probability for the rebinding diminishes according to Eq. (3) and Eq. (7).

\section{Two Model Parameters Determination}

In this section we present our results dealing with the determination of both unstressed offrate $\left(k_{r}^{0}\right)$ and the reactive compliance $\left(x_{\beta}\right)$ in the Bell model (Eqn. (4)) from the multiple bond force spectroscopy data.

Simulated Decay Without Rebinding-In this case we use the Levenberg-Marquardt non-linear least squares fitting program [31] to fit all the multiple bond rupture data for $\overline{\mathrm{LN}}$ vs $\overline{\mathrm{X}}$ to Eqn. (15) to calculate the Bell model parameters $\left(k_{r}^{0}, x_{\beta}\right)$. We applied this procedure to calculate the Bell model parameters $\left(k_{r}^{0}, x_{\beta}\right)$ at $N_{0}=200$ and $R_{f}=10^{5} \mathrm{pN} / \mathrm{s}$ and varying simulated unstressed off-rate from $k_{r}^{0}=1.6 \mathrm{~s}^{-1}$ to $k_{r}^{0}=8.6 \mathrm{~s}^{-1}$ for a fixed simulated reactive compliance $x_{\beta}=0.16 \AA$. Our findings are listed in Table VI. It is seen that the calculated Bell model parameters $\left(k_{r}^{0}, x_{\beta}\right)$ are very close to the simulated Bell model parameters $\left(k_{r}^{0}, x_{\beta}\right)$. In all cases the difference between the calculated parameters $\left(k_{r}^{0}, x_{\beta}\right)$ and the parameters prescribed in the simulations is less than $3.3 \%$.

Simulated Decay With Rebinding-To isolate the effectct of rebinding in the calculation of the Bell model parameters $\left(k_{r}^{0}, x_{\beta}\right)$ we generated data for $N(t)$ allowing for rebinding in the simulations, as described in the previous section. We were not able to develop an analytic two-parameter method that includes rebinding from Eq. (14). However, 
we can use the Levenberg-Marquardt non-linear least squares fitting program [31] to estimate the Bell model parameters $\left(k_{r}^{0}, x_{\beta}\right)$ from this data set. Note, this analysis, Eq.(15), neglects rebinding. The calculated Bell model parameters $\left(k_{r}^{0}, x_{\beta}\right)$ for loading rates $R_{f}=10^{3}$, $10^{4}, 10^{5}$, and $10^{6} \mathrm{pN} / \mathrm{s}$ are listed in Table VII. In all cases the difference between the calculated parameters $\left(k_{r}^{0}, x_{\beta}\right)$ and the parameters prescribed in the simulations is less than $2 \%$. These results demonstrate that effect of rebinding on the calculation of unstressed offrate is relatively small for the parameters used.

\section{CONSIDERATIONS FOR MEASUREMENT OF TRANSIENT BOND NUMBER}

Implementation of the analysis we have presented to determine the unstressed off-rate hinges on the measurement of $N(t)$, the transient history of the number of bonds during a multiple bond force spectroscopy experiment. Based on our simulation data we can discuss the attributes of an experimental method. Ideally, the method should be able to measure on the order of 100 bonds with a precision of one bond. The method must be able to resolve the decay of bond number as well. For the L-selectin-PSGL-1 bonds considered in this work, the time for the complete rupture of $N_{0}=200$ bonds at $R_{f}=10^{3}, 10^{4}, 10^{5}$, and $10^{6} \mathrm{pN} / \mathrm{s}$ are approximately $0.5,0.3,0.15$, and $0.05 \mathrm{~s}$ respectively. The corresponding applied forces at complete rupture are $500,3000,15000$, and $50000 \mathrm{pN}$, respectively. The time required for per unit rupture events are $2.5 \times 10^{-3}, 1.5 \times 10^{-3}, 0.75 \times 10^{-3}$, and $0.25 \times 10^{-3} \mathrm{~s}$, respectively. This implies that a temporal resolution of $10^{-3} \mathrm{~s}$ or less is needed to capture individual rupture events. Note that the temporal resolution depends on the unstressed offrate itself, and on the loading rate. While it is desirable to capture individual rupture events, our simulations and analysis method demonstrate that what is required to obtain the unstressed off-rate is a reasonably good fit to the transient history of bond number. Simulations of multiple bond force spectroscopy experiments like these can be used to design experimental techniques by predicting the effects of the temporal resolution and precision of $N(t)$ on the determination of the unstressed off-rate.

\section{CONCLUSION}

A novel method for calculating unstressed off-rate from multiple bonds rupture data is presented. The validity of the presented method is tested extensively. We successfully have shown that the calculated unstressed off-rate is in good agreement with the simulated unstressed off-rate at a given initial number of bonds and loading rate. However, our presented methodology is limited to the model equation of off-rates with monotonic dependency on the bond force such as Bell and Dembo models. Further using the Bell model we have shown that the present data analysis method can accurately determine the unstressed off-rates independent of the type of applied load when the initial number of bonds is large. Using the Bell model we demonstrated that calculated unstressed off-rate is in good agreement with the simulated unstressed off-rate at various loading rates for a fixed initial number of bonds and simulated unstressed off-rate. The same conclusion is reached when we varied simulated unstressed off-rate at a fixed initial number of bonds and loading rate. However, at a fixed loading rate and simulated unstressed off-rate the standard deviation in calculated unstressed off-rate increased progressively as we decrease initial number of bonds, implying that the precision of the present method for unstressed off-rate calculation increases with the initial number of bonds. It was also seen that including rebinding in the simulations using the Bell model with an unstressed on-rate of $150 \mathrm{~s}^{-1}$ and unstressed off-rate of $8.6 \mathrm{~s}^{-1}$ lead to small errors in the calculation of the off-rate and the reactive compliance when the data was analyzed using methods that assume no bond reformation. One particular feature of our approach is that with a simple model for multiple 
bond rupture we arrived at an extraction of unstressed off-rate which is both successful and effcient. This enables force spectroscopy experiments with large number of initial bonds and conditions with adhesion rates approaching one per touch. The development of an experimental technique that can measure the transient history of the number of bonds during multiple bond force spectroscopy experiments is warranted. This method would increase the throughput of the measurement of molecular bonds kinetics by reducing the number of touch and pull attempts required from $500-1000$ in single bond experiments to approximately 10 in multiple bond experiments.

Monte Carlo simulation of the rupture of multiple bonds between two PMNs is performed

We introduce a methodology to extract off-rate from multiple bond force spectroscopy

The methodology is tested extensively using both Bell and Dembo models

The precision for off-rate determination increases with the number of bonds

\section{Acknowledgments}

The authors would like to acknowledge the financial support provided by the National Institute of Health Grant RO1 AI063366. We thank the Pittsburgh Supercomputing Center for computational resources under DAC allocation MCB090098 and computational resources from HPCF at UMBC supported by the U.S. National Science Foundation through the MRI program (grant no. CNS-0821258) and the SCREMS program (grant no. DMS-0821311). The authors gratefully acknowledge helpful communication with David F.J. Tees.

\section{References}

1. Mustard JF, Packham MA, Kinlough-Rathbone RL, Perry DW, Regoeczi E. Blood. 1978; 52:453. [PubMed: 96891]

2. Albeldao SM. Lab. Invest. 1993; 68:4. [PubMed: 8423675]

3. Galkina E, Ley K. Arterioscl. Throm. Vasc. 2007; 27:2292.

4. Osborn L. Cell. 1990; 62:3. [PubMed: 2194672]

5. Lawrence MB, Springer TA. Cell. 1991; 65:859. [PubMed: 1710173]

6. Nicolson GL. Cancer Metastasis Rev. 1988; 7:143. [PubMed: 3293836]

7. Lasky LA. Science. 1992; 258:964. [PubMed: 1439808]

8. Simon SI, Green CE. Annu Rev Biomed Eng. 2005; 7:151. [PubMed: 16004569]

9. Erdmann T, Schwarz US. Eur. Phys. J. E. 2007; 22:123. [PubMed: 17347787]

10. Bell GI. Science. 1978; 200:618. [PubMed: 347575]

11. Sulchek TA. PNAS. 2005; 102:16638. [PubMed: 16269547]

12. Sulchek TA, Friddle RW, Noy A. Biophys. J. 2006; 90:4686. [PubMed: 16581843]

13. Yipp BG, et al. Blood. 2000; 96:2292. [PubMed: 10979979]

14. Casares S, et al. J. Exp. Med. 1999; 190:543. [PubMed: 10449525]

15. Holliger P, Prospero T, Winter G. PNAS. 1993; 90:6444. [PubMed: 8341653]

16. Zhang Y, Sun G, Lü S, Li N, Long M. Biophys. J. 2008; 95:5439. [PubMed: 18757559]

17. Erdmann T, Pierrat S, Nassoy P, Schwarz US. EPL. 2008; 81:48001.

18. Williams PM. Anal. Chim. Acta. 2003; 479:107.

19. Gu C, Kirkpatrick A, Ray C, Guo S, Akhremitchev BB. J. Phys. Chem. C. 2008; 112:5085.

20. Dammer U, et al. Biophys. J. 1996; 70:2437. [PubMed: 9172770]

21. Williams JM, Han TJ, Beebe TP TP. Langmuir. 1996; 12:1291.

22. Allen S, et al. Biochemistry. 1997; 36:7457. [PubMed: 9200694]

23. Hukkanen EJ, Wieland JA, Gewirth A, Leckband DE, Braatz RD. Biophys. J. 2005; 89:3434. [PubMed: 16100278] 
24. Gupta VK, Sraj IA, Konstantopoulos K, Eggleton CD. Biomechan. Model. Mechanobiol. 2010; 9:613.

25. Gupta VK, Eggleton CD. Phys. Rev. E. 2011; 84:011912.

26. Hammer DA, Apte SM. Biophys. J. 1992; 63:35. [PubMed: 1384734]

27. Dembo, M. Vol. 24 of series: Lectures on Mathematics in the Life Sciences, Some Mathematical Problems in Biology. Providence, RI: American Mathematical Society; 1994. On peeling an adherent cell from a surface; p. 51-77.

28. Erdmann T, Schwarz US. Europhys. Lett. 2004; 66:603.

29. Seifert U. Phys. Rev. Lett. 2000; 84:2750. [PubMed: 11017316]

30. Tees DF, Waugh RE, Hammer DA. Biophys. J. 2001; 80:668. [PubMed: 11159435]

31. Press, WH.; Teukolsky, SA.; Vetterling, WT.; Flanery, BP. Numerical Recipes C. New York: Cambridge University Press; 1997. 


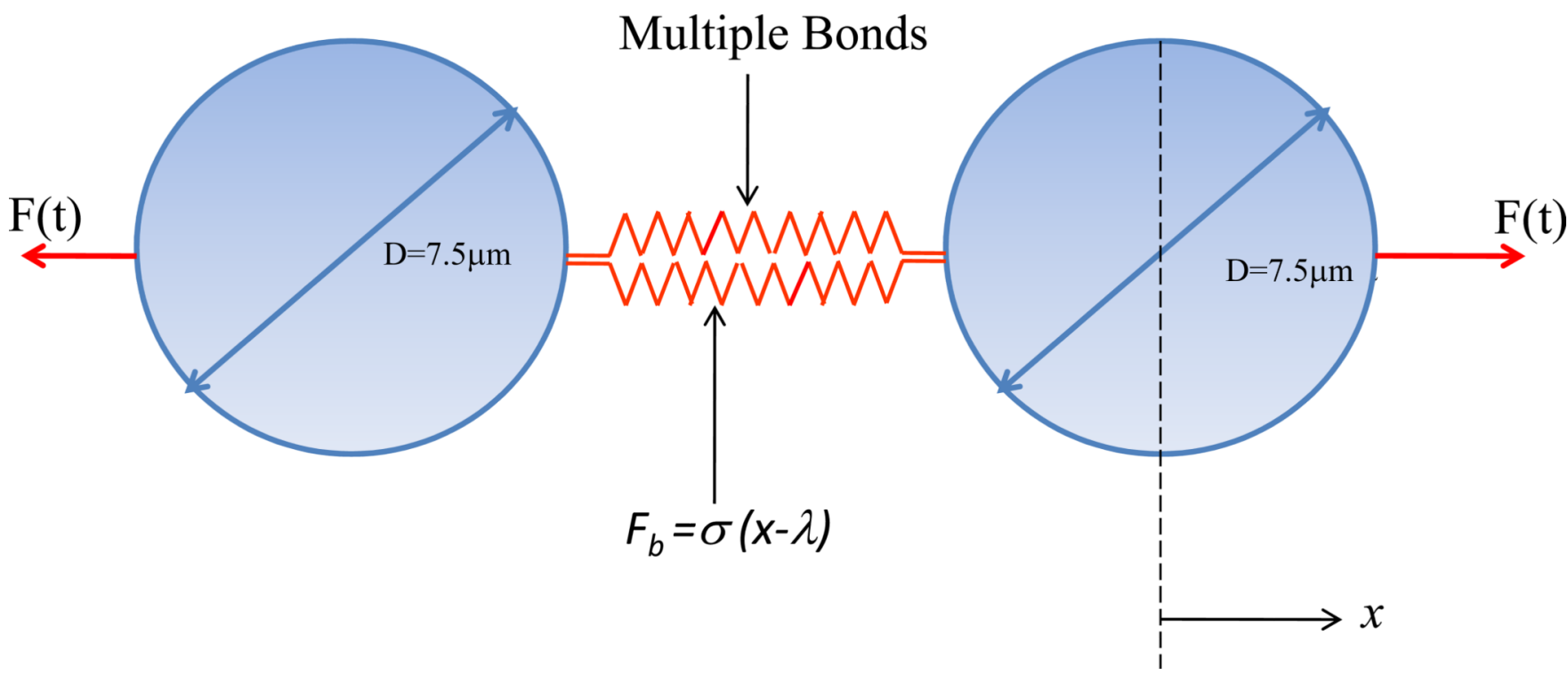

FIG. 1.

Schematic of two PMN cells detachment simulation (not drawn to scale) through binding between PSGL-1 (receptor) and L-selectin (ligand) molecules concentrated on the tip of PMN microvillus. 

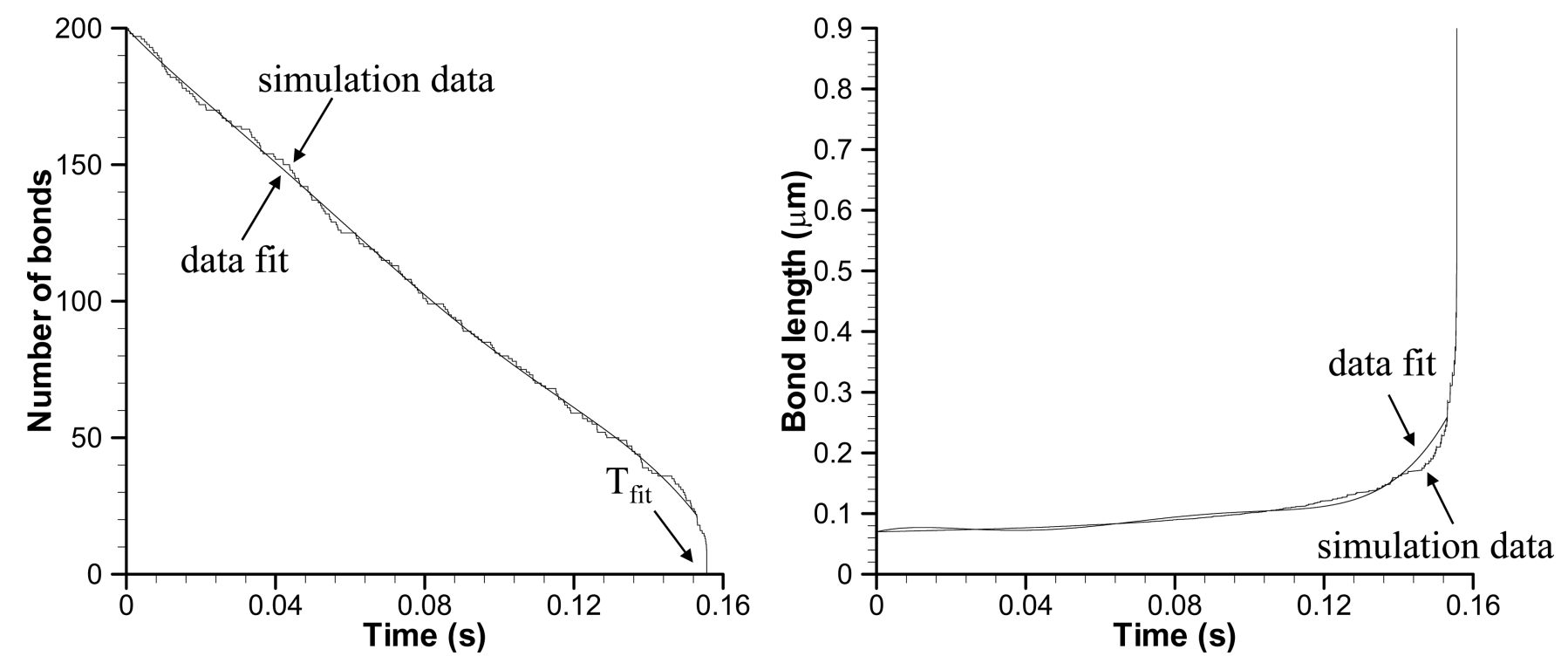

FIG. 2.

Time variation of (a) number of bonds (b) bond length obtained from micromechanical model simulation using Bell model at $R_{f}=10^{5} \mathrm{pN} / \mathrm{s}, N_{0}=200$, and $k_{r}^{0}=8.6 \mathrm{~s}^{-1}$. Corresponding data fits $N(t)=A t^{5}+B t^{4}+C t^{3}+D t^{2}+E t+N_{0}$ with $A=-6426810, B=$ 2153400, $C=-243128, D=11472.3, E=-1422.04, N_{0}=200$ and $x(t)=a t^{5}+b t^{4}+c t^{3}+$ $d t^{2}+e t+x_{0}$ with $a=53903.7, b=-17545.5, c=1994.05, d=-88.4963, e=1.39597, x_{0}=$ $0.07 \mu \mathrm{m}$ are also shown in (a) and (b), respectively. 


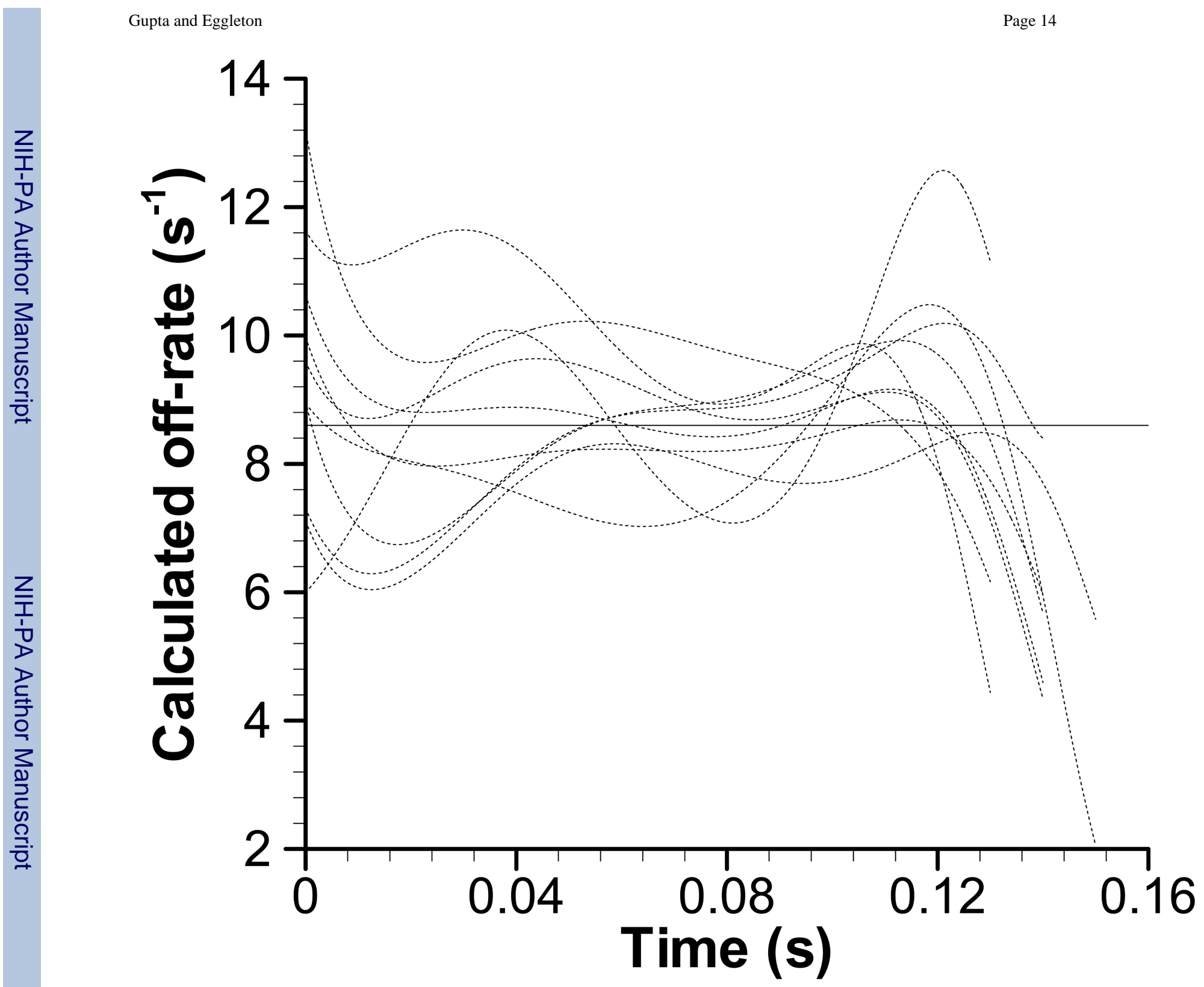

FIG. 3.

Time variation of calculated unstressed off-rate (dashed lines) obtained from micromechanical model simulation using the Bell model at $R_{f}=10^{5} \mathrm{pN} / \mathrm{s}, N_{0}=200$, and $k_{r}^{0}=8.6 \mathrm{~s}^{-1}$. Solid line represents simulated unstressed off-rate $\left(k_{r}^{0}=8.6 \mathrm{~s}^{-1}\right)$. 


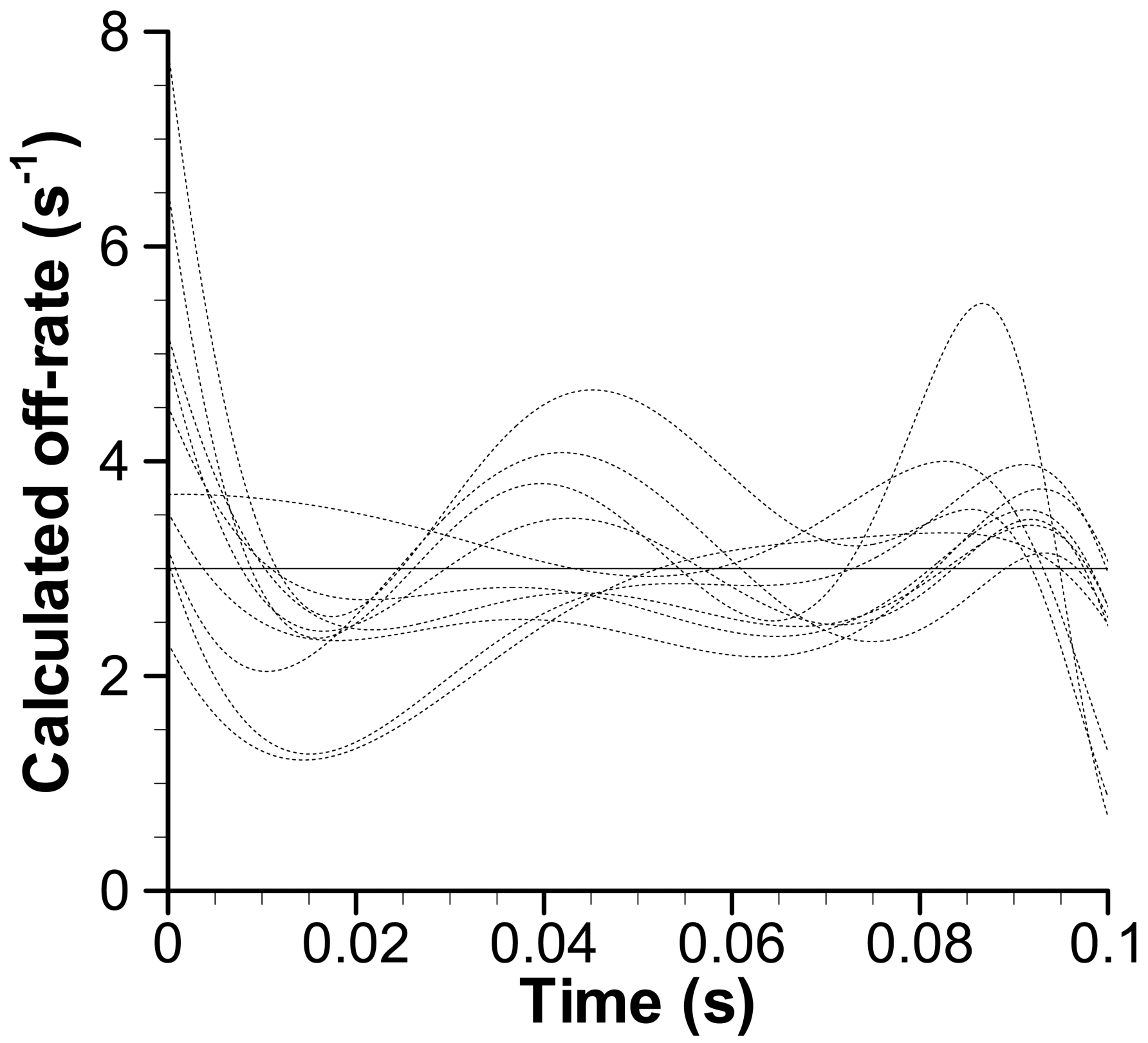

FIG. 4.

Time variation of calculated unstressed off-rate (dashed lines) obtained from micromechanical model simulation using the Dembo model at $R_{f}=10^{5} \mathrm{pN} / \mathrm{s}, N_{0}=200$, and $k_{r}^{0}=3.0 \mathrm{~s}^{-1}$. Solid line represents simulated unstressed off-rate $\left(k_{r}^{0}=3.0 \mathrm{~s}^{-1}\right)$. 


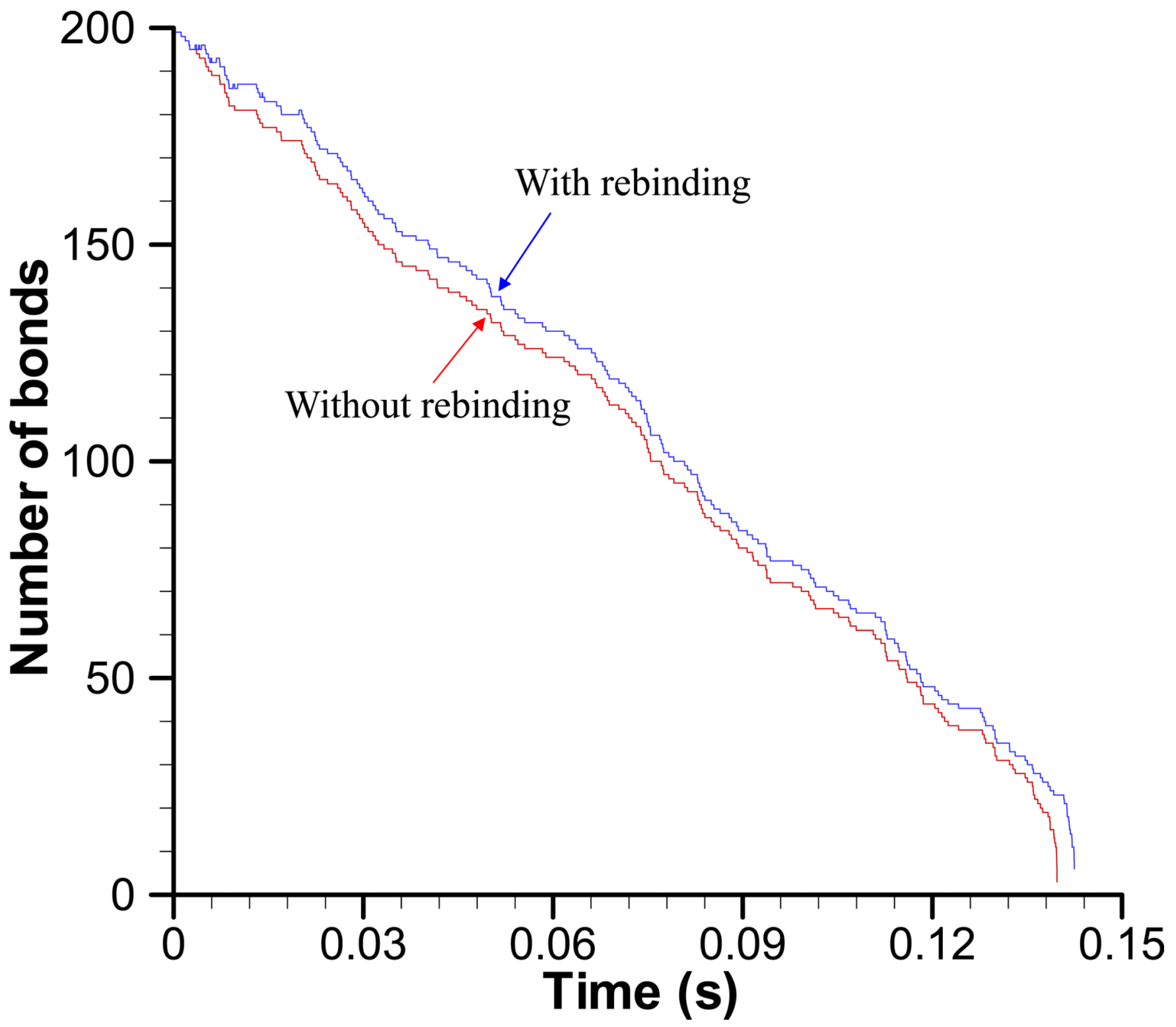

FIG. 5.

A typical variation of number of bonds with (blue lines) and without (red lines) rebinding obtained from micromechanical model simulation using Bell model at $R_{f}=10^{5} \mathrm{pN} / \mathrm{s}, N_{0}=$ $200, k_{f}^{0}=150.0 \mathrm{~s}^{-1}$, and $k_{r}^{0}=8.6 \mathrm{~s}^{-1}$. 
TABLE I

Parameter values used in the model

\begin{tabular}{|c|c|c|}
\hline Parameter & Definition & Value \\
\hline$R$ & PMN radius & $3.75 \mu \mathrm{m}$ \\
\hline$\lambda$ & Receptor-ligand bond length & $0.07 \mu \mathrm{m}$ \\
\hline$k_{f}^{0}$ & Unstressed on-rate (Bell) & $150 \mathrm{~s}^{-1}$ \\
\hline$k_{f}^{0}$ & Unstressed on-rate (Dembo) & $3 \mathrm{~s}^{-1}$ \\
\hline$\sigma$ & & \\
\hline$\sigma_{\mathrm{ts}}$ & Transition state spring constant (Dembo) & $3.96 \mathrm{dyn} / \mathrm{cm}$ \\
\hline$x_{\beta}$ & Reactive compliance (Bell) & $0.16 \AA$ \\
\hline$\mu$ & Fluid viscosity & $0.8 \mathrm{cP}$ \\
\hline$\rho$ & Fluid density & $1 \mathrm{~g} / \mathrm{cc}$ \\
\hline$T$ & Temperature & $310 \mathrm{~K}$ \\
\hline
\end{tabular}


TABLE II

Bell off-rate calculation for L-selectin-PSGL-1 interaction without rebinding and $F=R_{f} t$.

\begin{tabular}{|c|c|c|c|}
\hline $\boldsymbol{R}_{\boldsymbol{f}}(\mathbf{p N} / \mathbf{s})$ & $\boldsymbol{N}_{\mathbf{0}}$ & Simulated off-rate, $k_{\boldsymbol{r}\left(\mathbf{s}^{\mathbf{1}}\right)}$ & Calculated off-rate $\pm \mathbf{S D}\left(\mathbf{s}^{\mathbf{- 1}}\right)$ \\
\hline $10^{3}$ & 200 & 8.6 & $8.74 \pm 0.56$ \\
\hline $10^{4}$ & 200 & 8.6 & $8.67 \pm 0.45$ \\
\hline $10^{5}$ & 200 & 8.6 & $8.65 \pm 0.67$ \\
\hline $10^{6}$ & 200 & 8.6 & $8.73 \pm 0.84$ \\
\hline $10^{5}$ & 200 & 1.6 & $1.60 \pm 0.09$ \\
\hline $10^{5}$ & 200 & 3.6 & $3.70 \pm 0.22$ \\
\hline $10^{5}$ & 200 & 5.6 & $5.48 \pm 0.30$ \\
\hline $10^{5}$ & 200 & 8.6 & $8.65 \pm 0.67$ \\
\hline $10^{5}$ & 500 & 8.6 & $8.71 \pm 0.23$ \\
\hline $10^{5}$ & 200 & 8.6 & $8.65 \pm 0.67$ \\
\hline $10^{5}$ & 100 & 8.6 & $8.52 \pm 0.76$ \\
\hline $10^{5}$ & 50 & 8.6 & $8.45 \pm 0.84$ \\
\hline
\end{tabular}




\section{TABLE III}

Bell off-rate calculation for L-selectin-PSGL-1 interaction without rebinding and $F=F_{0}\left[1-\exp \left(-\frac{R_{f} t}{F_{0}}\right)\right]$.

\begin{tabular}{|c|c|c|c|c|}
\hline $\boldsymbol{R}_{\boldsymbol{f}}(\mathbf{p N} / \mathbf{s})$ & $\boldsymbol{F}_{\mathbf{0}}(\mathbf{p N})$ & $\boldsymbol{N}_{\mathbf{0}}$ & Simulated off-rate, $k_{\boldsymbol{r}\left(\mathbf{s}^{\mathbf{- 1}}\right)}$ & \\
\hline $10^{5}$ & 200 & 200 & 8.6 & $8.57 \pm 0.26$ \\
\hline $10^{4}$ & 250 & 200 & 8.6 & $8.54 \pm 0.29$ \\
\hline $10^{3}$ & 200 & 200 & 8.6 & $8.62 \pm 0.31$ \\
\hline $10^{3}$ & 150 & 200 & 8.6 & $8.68 \pm 0.23$ \\
\hline
\end{tabular}




\section{TABLE IV}

Dembo off-rate calculation for L-selectin-PSGL-1 interaction without rebinding and $F=R_{f} t$.

\begin{tabular}{|c|c|c|c|}
\hline $\boldsymbol{R}_{\boldsymbol{f}}(\mathbf{p N} / \mathbf{s})$ & $\boldsymbol{N}_{\mathbf{0}}$ & Simulated off-rate, $k_{\boldsymbol{r}}^{0}\left(\mathbf{s}^{\mathbf{- 1}}\right)$ & Calculated off-rate $\pm \mathbf{S D}\left(\mathbf{s}^{\mathbf{- 1}}\right)$ \\
\hline $10^{3}$ & 200 & 3.00 & $3.08 \pm 0.26$ \\
\hline $10^{4}$ & 200 & 3.00 & $3.02 \pm 0.16$ \\
\hline $10^{5}$ & 200 & 3.00 & $3.01 \pm 0.39$ \\
\hline $10^{6}$ & 200 & 3.00 & $3.08 \pm 0.53$ \\
\hline $10^{5}$ & 200 & 1.00 & $0.98 \pm 0.10$ \\
\hline $10^{5}$ & 200 & 3.00 & $3.01 \pm 0.39$ \\
\hline $10^{5}$ & 200 & 7.00 & $7.00 \pm 0.88$ \\
\hline $10^{5}$ & 200 & 10.00 & $9.95 \pm 1.20$ \\
\hline $10^{5}$ & 500 & 3.00 & $3.05 \pm 0.17$ \\
\hline $10^{5}$ & 200 & 3.00 & $3.01 \pm 0.39$ \\
\hline $10^{5}$ & 100 & 3.00 & $2.94 \pm 0.70$ \\
\hline $10^{5}$ & 50 & 3.00 & $2.88 \pm 0.79$ \\
\hline
\end{tabular}


TABLE V

Bell off-rate calculation for L-selectin-PSGL-1 interaction with rebinding and $F=R_{f} t$.

\begin{tabular}{|c|c|c|c|}
\hline $\boldsymbol{R}_{\boldsymbol{f}}(\mathbf{p N} / \mathbf{s})$ & $\boldsymbol{N}_{\mathbf{0}}$ & Simulated off-rate, $k_{\boldsymbol{r}\left(\mathbf{s}^{\mathbf{- 1}}\right)}$ & Calculated off-rate $\pm \mathbf{S D}\left(\mathbf{s}^{\mathbf{- 1}}\right)$ \\
\hline $10^{3}$ & 200 & 8.6 & $8.69 \pm 0.38$ \\
\hline $10^{4}$ & 200 & 8.6 & $8.71 \pm 0.29$ \\
\hline $10^{5}$ & 200 & 8.6 & $8.60 \pm 0.22$ \\
\hline $10^{6}$ & 200 & 8.6 & $8.67 \pm 0.34$ \\
\hline
\end{tabular}


